\title{
Ein finanzpolitisches Notfallprogramm zur Rettung des sozialen Europas
}

\author{
Das soziale Europa ist durch das in Europa vorherrschende und in den wirtschafts- \\ politischen Institutionen der Europäischen Union (EU) verankerte makroökonomische \\ Paradigma im Kern bedroht. Da weder ein Wandel des Paradigmas noch der Institutionen \\ kurzfristig realistisch erscheint, stellt sich die Frage nach zeitnah umsetzbaren Notfall- \\ maßnahmen innerhalb des bestehenden institutionellen Rahmens. Ist ein Ende der \\ Austeritätspolitik in Europa möglich? Ist es möglich, die Finanzpolitik als makroöko- \\ nomisches Instrument zu reaktivieren und einen kräftigen expansiven Impuls zu setzen?
}

ACHIM TRUGER

\section{Einleitung}

Das soziale Europa ist spätestens seit Beginn der Eurokrise in seinem Kern bedroht. Diese Bedrohung geht von der Ausrichtung der makroökonomischen Politik auf Austerität und von der Durchführung sogenannter Strukturreformen aus, zu denen die Deregulierung des Arbeitsmarktes, die Schwächung von Arbeitnehmerrechten und der Abbau des Sozialstaats gehören. Diese Politik hat Südeuropa in eine wirtschaftliche Depression und die EU in eine tiefe soziale und politische Krise geführt.

Aus keynesianischer Sicht sind die ökonomischen Ursachen der Krise relativ leicht zu identifizieren. Die europäische Wirtschafts-, Sozial- und Arbeitsmarktpolitik hat falsch auf die Krise reagiert. Anstatt angesichts des offenkundigen Versagens deregulierter Märkte auf Reregulierung und expansive makroökonomische Politik zu setzen, treibt sie Deregulierung und Sozialabbau voran. Das einzige noch wirksame makroökonomische Instrument, die Finanzpolitik, wurde durch die Fesseln des Stabilitäts- und Wachstumspaktes (SWP) in eine krisenverschärfende Austeritätspolitik getrieben. Ursache der falschen Reaktion sind damit das europäische wirtschaftspolitische Regelwerk und das dahinter stehende makroökonomische Paradigma, das auf Deregulierung und einen weitgehenden Verzicht auf makroökonomische (Nachfrage-)Politik drängt.
Die naheliegende Schlussfolgerung besteht in der Aufgabe des herrschenden ökonomischen Paradigmas und in einer tief greifenden Reform des wirtschaftspolitischen Regelwerks im Sinne einer koordinierten makroökonomischen Politik (vgl. schon Hein et al. 2004; Hein et al. 2012). Da ein solcher tief greifender Wandel kurzfristig politisch unrealistisch ist, lotet der vorliegende Beitrag die Möglichkeiten eines wesentlich weniger ambitionierten fiskalpolitischen Notfallprogramms zur Rettung des sozialen Europas aus. Wenn es gelingt, der verheerenden Austeritätspolitik ein Ende zu bereiten und eine expansive Fiskalpolitik einzuleiten, könnte die ökonomische Krise beendet und über mehr Wachstum und Beschäftigung auch zu einer erheblichen Linderung der sozialen Misere beigetragen werden. Da kurzfristig Änderungen des fiskalpolitischen Regelwerks wenig realistisch erscheinen, stellt sich die Frage, ob ein solches expansives Notfallprogramm innerhalb des bestehenden Regelwerks umgesetzt werden könnte. Gerade angesichts der Tatsache, dass die Regeln erst kürzlich erheblich verschärft worden sind (Europäische Kommission 2013, S. 13ff.), ist zudem zu fragen, ob in diesem Rahmen noch ein hinreichend starker expansiver Fiskalimpuls erzielt werden kann.

Der vorliegende Beitrag vertritt die Auffassung, dass ein solcher Impuls tatsächlich noch möglich ist - vorausgesetzt, dass die EU-Kommission willens ist, die technisch-statistischen und interpretatorischen Spielräume, die die zentralen unklaren Konzepte des bestehenden Regelwerks lassen, 
zu nutzen. Um dies zu zeigen, verdeutlicht Abschnitt 2 zunächst, dass ein soziales Europa mit dem herrschenden makroökonomischen Paradigma bzw. seiner Verkörperung in den EU-Institutionen nicht vereinbar ist. Im Anschluss hieran werden das Ausmaß und die Auswirkungen der bisherigen Austeritätspolitik dargelegt (3). Abschnitt 4 behandelt die jüngsten Pläne der neuen EU-Kommission und erläutert, dass sie für einen spürbaren positiven Impuls kaum ausreichen werden. Der darauffolgende Abschnitt skizziert, wie eine weitreichendere Nutzung der existierenden Spielräume aussehen könnte (5). Abschnitt 6 zieht ein kurzes Fazit.

\section{Die herrschende Makroökonomik in der EU als Feind von Sozialstaat und Wachstum}

Die Makroökonomik ist spätestens seit der General Theory von John Maynard Keynes durch die Konkurrenz unterschiedlicher Paradigmen geprägt (Snowdon/Vane 2005; Heine/Herr 2013). Seit den 1970er Jahren wurde der Keynesianismus als dominierendes Paradigma zunächst von Monetarismus und Neuklassik und schließlich in den 1990er und 2000er Jahren vom Neu-Keynesianismus, zuletzt im Gewande des sogenannten Neuen Konsens, abgelöst (Arestis 2011; Truger 2003). Allen diesen theoretischen Richtungen ist gemeinsam, dass sie grundsätzlich mittel- bis langfristig Arbeitslosigkeit allein als Ergebnis von Abweichungen vom Ideal des neoklassischen, unregulierten Arbeitsmarktes auffassen. Arbeitsmarktregulierungen wie etwa der Kündigungsschutz, sonstige Arbeitsschutzgesetze, Mitbestimmung oder ein hoher gewerkschaftlicher Organisationsgrad führen demzufolge über die Stärkung der Verhandlungsmacht der Arbeitnehmerseite und die damit verbundenen zu hohen Löhne zu Arbeitslosigkeit. Dasselbe gelte für die Institutionen des Sozialstaates: Die Arbeitslosenversicherung und die Sozialhilfe erhöhten den „Anspruchslohn“; lohnbezogene Steuern und Abgaben, der sogenannte Steuerkeil, führten zu höheren kompensierenden Lohnforderungen und mithin ebenfalls zu Arbeitslosigkeit (OECD 1994). Je stärker der Regulierungsgrad und die sozialstaatlichen Institutionen, desto höher sei die institutionelle Verkrustung und damit das Niveau an „struktureller" Arbeitslosigkeit.

Makroökonomische Interventionen durch die Finanzoder Geldpolitik können demzufolge allenfalls kurzfristig Produktion und Beschäftigung beeinflussen, langfristig hingen diese von den "strukturellen“ Charakteristika des Arbeitsmarktes und des Sozialstaates ab. In der neueren Mainstream-Variante der Makroökonomik ist es allein die Geldpolitik, die kurzfristig mit dem Ziel der Inflationssteuerung über die Zinssetzung Nachfrage und damit Produktion sowie Beschäftigung steuert, während die Finanzpoli- tik an Bedeutung verliert. Eine expansive Finanzpolitik wird für wirkungslos gehalten, weil die positiven Impulse staatlicher Mehrausgaben oder Steuersenkungen durch erwartungsbedingte Verhaltensanpassungen von privaten Haushalten und Unternehmen genau kompensiert würden: Bei gestiegener Staatsverschuldung erwarteten die privaten Haushalte zukünftige Steuererhöhungen und senkten daher ihren Konsum, um Geld für die Zahlung der Steuern zurückzulegen. Die Unternehmen erwarteten steigende Zinsen und reduzierten daher ihre Investitionen.

Für die Wirtschaftspolitik bedeutet dies, dass die Finanzpolitik als makroökonomisches Instrument ausscheidet und sich lediglich auf das Ziel ausgeglichener Haushalte konzentrieren solle (Arestis/Sawyer 2003). Der Geldpolitik kommt die kurzfristige Aufgabe der Nachfragesteuerung zum Zwecke der Inflationssteuerung und -bekämpfung zu. Eine dauerhafte, „strukturelle“ Verbesserung der Wirtschaftslage und der Beschäftigung ist aus dieser Sicht nur über die Deregulierung des Arbeitsmarkes, die Schwächung von Gewerkschaften und den Abbau des Sozialstaates zu erreichen.

Genau darin besteht die grundsätzliche Feindschaft des herrschenden makroökonomischen Paradigmas gegenüber einem sozialen Europa. Dies wäre möglicherweise anders zu beurteilen, wenn die Umsetzung der wirtschaftspolitischen Empfehlungen tatsächlich zu höherem Wachstum und einer geringeren Arbeitslosigkeit führen würde. Allerdings ist genau dies weder theoretisch noch empirisch plausibel. Aus theoretischer Sicht lässt sich zeigen, dass eine konsequente Formulierung des zugrunde gelegten Modells letztlich sogar zu (post-)keynesianischen Ergebnissen führt (Lavoie 2009; Hein/Stockhammer 2011). Zudem lässt sich a priori nicht sagen, ob die „strukturellen“ Hemmnisse beschäftigungssenkend oder -steigernd sind (Truger/Hein 2003). Aus empirischer Sicht ist weder die konstatierte Wirkungslosigkeit der Fiskalpolitik (vgl. Abschnitt 3) noch die behauptete Beschäftigungsfeindlichkeit von Arbeitsmarktregulierung und Sozialstaat aufrecht zu erhalten (Baker et al. 2004; Truger/Hein 2003).

Dies ändert jedoch nichts daran, dass die wirtschaftspolitischen Institutionen der EU, insbesondere aber des Euroraums, ganz im Sinne der herrschenden Makroökonomik konstruiert sind (vgl. kritisch Hein/Truger 2004 sowie zustimmend Issing 2002). Die Finanzpolitik wurde durch den SWP mit der Verpflichtung auf strukturell und mittelfristig nahezu ausgeglichene Haushalte entmachtet, während die Geldpolitik auf das Ziel der Preisniveaustabilität verpflichtet wurde. Zwar finden sich im Rahmen des sogenannten „Köln-Prozesses“ mit dem makroökonomischen Dialog auch andere Ansätze, jedoch sind diese marginalisiert worden. Im Bereich der Arbeitsmarktpolitik dominieren seit den beschäftigungspolitischen und den wirtschaftspolitischen Leitlinien ebenfalls die „strukturellen“ Reformempfehlungen von Deregulierung und Sozialabbau (Hein/Niechoj 2007). Auf die Spitze getrieben wurde dieses Politikmodell schließlich durch die drastische Austeritäts- 
politik in den Staaten der Peripherie im Zuge der Eurokrise (vgl. Abschnitt 3) sowie die in diesem Zusammenhang von der Troika oder der EU-Kommission sowie dem Europäischen Rat verordnete radikale „strukturelle Reformpolitik" in den Krisenstaaten (Hermann/Hinrichs 2012; Darvas et al. 2014)

\section{Austeritätspolitik in Europa als Scheitern der herrschenden Makroökonomik}

Die langfristige Unvereinbarkeit der herrschenden Makroökonomik mit einem sozialem Europa wurde aufgrund günstiger ökonomischer Rahmenbedingungen noch nicht unmittelbar nach der Einführung des Euro, sondern erst nach dem Einsetzen der globalen Finanz- und Wirtschaftskrise im Jahr 2007 sichtbar. Zwar hatten die restriktive Finanzpolitik und die Disinflationspolitik zur Einhaltung der Maastricht-Kriterien im Vorfeld der Euro-Einführung bereits zu deutlichen Wachstums- und Beschäftigungsverlusten geführt (Hein/Truger 2007). Allerdings war im EU- und im Euroraum-Durchschnitt die makroökonomische Performance vor dem Einsetzen der Finanz- und Wirtschaftskrise noch halbwegs zufriedenstellend. Dies lag daran, dass die - insbesondere fiskalpolitischen - makroökonomischen Fesseln des europäischen wirtschaftspolitischen Regelwerks in ihrer negativen Wirkung durch außergewöhnliche vorübergehende positive Faktoren übertüncht wurden. Dazu zählten vor allem die Konvergenz in die Richtung des niedrigen deutschen Zinsniveaus, ein günstiges außenwirtschaftliches Umfeld und der finanzmarkt- und immobiliengetriebene Konsumboom in einigen Volkswirtschaften.

Dies änderte sich jedoch jäh mit der globalen Finanzund Wirtschaftskrise und der in der Folge auftretenden Eurokrise. Nach einer kurzen expansiven Phase während der globalen Finanz- und Wirtschaftskrise war die Finanzpolitik in der Eurozone wegen der strikten Interpretation des SWP extrem restriktiv ausgerichtet. Vor allem die sogenannten Peripherie-Länder (Griechenland, Irland, Portugal und Spanien), deren Staatsschuldpapiere auf den Finanzmärkten spekulativen Attacken zum Opfer fallen, wurden unter den Rettungsprogrammen und/oder von EUKommission und Europäischem Rat unter strikter Auslegung und Verschärfung der Regeln des SWP zur Umsetzung harter Austeritätsprogramme gezwungen (Blyth 2013; Truger 2013).

Abbildung 1 zeigt den gesamtstaatlichen strukturellen Primärsaldo für die Eurozone (zwölf „alte” Mitgliedsländer), die Peripherie sowie Deutschland und Frankreich von 1999 bis 2013. Die jährliche Veränderung dieser Größe ist ein trotz seiner konzeptionellen Schwächen häufig verwendeter - Indikator für die Ausrichtung der Fiskalpolitik, d. h. die
ABB.

\section{Gesamtstaatlicher struktureller Primärsaldo* in der Eurozone, der Peripherie und ausgewählten Ländern, 1999 - 2013}

Angaben in Prozent des BIP

- Eurozone (12 Länder)

- Peripherie (EL, ES, PT, IE**)

$=-=$ Deutschland

-.- Frankreich

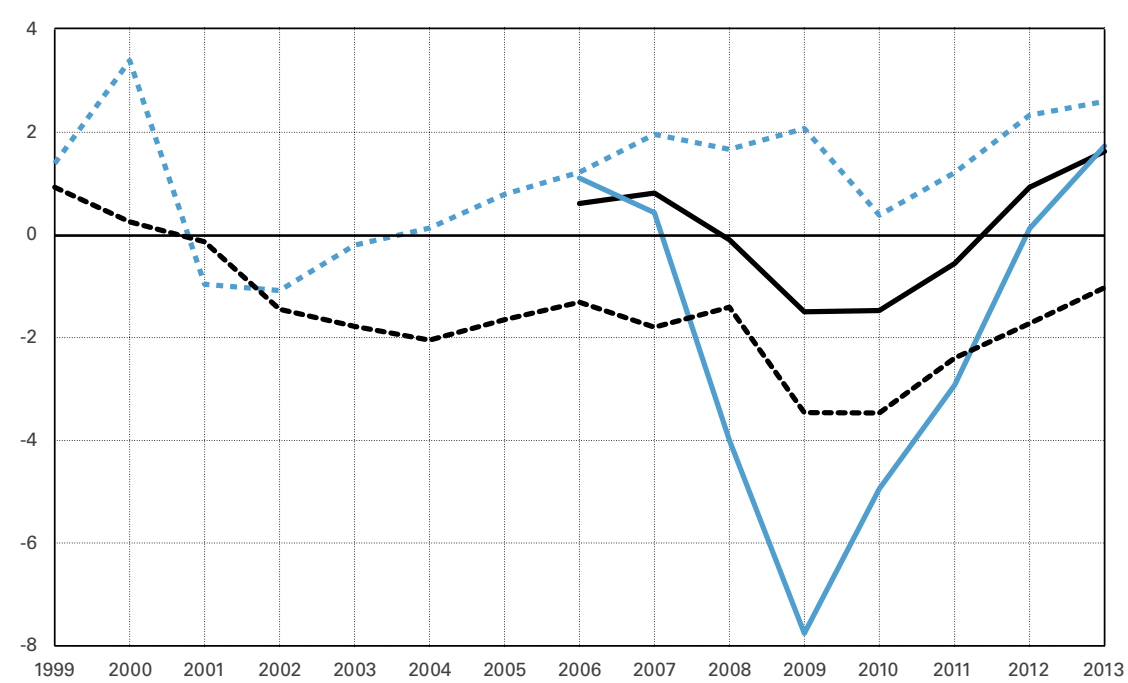

* Der Primärsaldo ist die Differenz von Staatseinnahmen und Staatsausgaben unter Ausschluss von Zinsausgaben; der strukturelle Primärsaldo entsteht durch die Bereinigung um konjunkturelle Effekte. ${ }^{* *} \mathrm{EL}=$ Griechenland, $\mathrm{ES}=$ Spanien, $\mathrm{PT}=$ Portugal, IE = Irland.

Quelle: Europäische Kommission (2014a). Berechnungen und Darstellung des Autors.

diskretionären Änderungen in der Fiskalpolitik. Offensichtlich verbesserte sich dieser Saldo seit der Krise überall deutlich, d. h. die strukturellen Defizite wurden zurückgeführt. Das Ausmaß der Restriktion für die Eurozone insgesamt lag zwischen 2010 und 2013 bei etwa $3 \%$ des Bruttoinlandproduktes (BIP). In der Peripherie insgesamt lag es von 2009 bis 2013 sogar bei annähernd $10 \%$ des BIP.

Hinzu kommt, dass es mittlerweile weithin akzeptiert ist, dass die Veränderung des strukturellen Saldos ein problematischer Indikator für die Ausrichtung der Finanzpolitik ist. Das Ausmaß der fiskalischen Restriktion wird hierdurch in Krisenphasen erheblich unterschätzt. Der strukturelle Primärsaldo wird berechnet, indem man den tatsächlichen Primärsaldo konjunkturell bereinigt und Einmaleffekte (Privatisierungserlöse etc.) herausrechnet. Die üblichen Konjunkturbereinigungsmethoden unterschätzen das Ausmaß von Konjunkturschwankungen und führen zu einer prozyklischen Politik, wenn sie zum Maßstab der Fiskalpolitik gemacht werden. Insbesondere die Methode der EU-Kommission hat sich diesbezüglich als besonders anfällig erwiesen, weil das von ihr errechnete Produktionspotenzial stark von der aktuellen Konjunkturlage und besonders von der Arbeitslosenquote beeinflusst wird (Klär 2014; Truger/Will 2012). In ökonomischen Abschwungphasen - besonders in kräftigen und lang anhaltenden, wie sie 
in Europa zu beobachten sind - wird das Produktionspotenzial schnell und stark nach unten revidiert. Insbesondere die Anpassung der zugrunde gelegten strukturellen Arbeitslosenquote an die tatsächliche Arbeitslosenquote erfolgt dabei sehr schnell. In dieser Interpretation wären laut Europäischer Kommission (2014a) z. B. im Jahr 2010 annähernd $93 \%$ der Arbeitslosen im Euroraum strukturell arbeitslos gewesen; die Arbeitslosenquote hätte sich von 10,1\% durch konjunkturpolitische Maßnahmen lediglich auf 9,4\% senken lassen. Jede weitere Senkung hätte der herrschenden Lehre zufolge bereits einen dauerhaften Inflationsschub ausgelöst. Angesichts der Tatsache, dass der Euroraum 2010 mit der tiefsten Konjunkturkrise seit dem Zweiten Weltkrieg zu kämpfen hatte, erscheint eine solche Diagnose kaum plausibel.

Die Abwärtsrevision des Produktionspotenzials ist kein akademisches Problem, sondern hat konkrete gravierende Folgen für die errechneten strukturellen Defizite und die ermittelten Konsolidierungsanstrengungen (Heimberger 2014). Letztere werden üblicherweise zu gering angesetzt, weil ein größerer Teil des Defizits als strukturell verbucht wird, obwohl er möglicherweise lediglich durch den Konjunktureinbruch hervorgerufen wurde (siehe dazu auch Abschnitt 5).

Die EU-Kommission hat längst zugeben müssen, dass die von ihr auf Basis der Veränderung der strukturellen Defizite geschätzten Konsolidierungsbemühungen die tatsächlichen Anstrengungen erheblich unterschätzen und zieht daher mittlerweile bereits zusätzliche Indikatoren heran (Carnot/de Castro 2015). Wenn man die Ergebnisse von Carnot und de Castro (2015, S. 10) verwendet, dann ergibt sich, dass die in Abbildung 1 dargestellten Konsolidierungsanstrengungen das wahre Ausmaß im Falle Portugals um $20 \%$, Irlands um $45 \%$, Spaniens um fast $75 \%$ und das Griechenlands sogar um fast $90 \%$ zu gering ausweisen. Damit läge das tatsächliche Konsolidierungsvolumen in der Peripherie von 2009 bis 2013 bei insgesamt $16 \%$ des BIP - und nicht bei "nur" $10 \%$ des BIP, wie in Abbildung 1 ausgewiesen (siehe auch Darvas et al. 2014, S. 10ff.).

Die potenziellen ökonomischen Konsequenzen der Austeritätspolitik können leicht mithilfe des Fiskalmultiplikators veranschaulicht werden. Wenn man das kumulierte Konsolidierungsvolumen mit dem Multiplikator multipliziert, erhält man eine grobe Schätzung dafür, wie stark sich das BIP durch die Konsolidierung vermindert hat. Die neuesten Schätzungen legen nahe, dass der Multiplikator relativ hohe Werte von durchaus deutlich über Eins annimmt - insbesondere in der gegenwärtigen Lage in der Eurozone, in der die Geldpolitik die Nullzinsgrenze erreicht hat und in der fast alle Länder gleichzeitig Sparpolitik betreiben (Gechert 2015). Wenn man Multiplikatoren in dieser Größenordnung auf die angesprochenen Konsolidierungsmaßnahmen anwendet, betragen die Auswirkungen der Austeritätspolitik für die gesamte Peripherie im Jahr 2013 mehr als $16 \%$ des BIP, das BIP in der Peripherie hätte ohne diese Politik also mehr als $16 \%$ höher sein können. Tatsächlich lässt sich eine starke negative Korrelation zwischen der Höhe der Konsolidierungsanstrengungen und dem Wirtschaftswachstum in den Ländern der Eurozone feststellen (Truger 2014). Die Länder mit der stärksten Restriktion schnitten beim BIP-Wachstum tendenziell am schlechtesten ab.

Wie Darvas et al. (2014) zeigen, waren nicht nur die ökonomischen, sondern auch die sozialen Kosten der Austeritätspolitik im Sinne eines Anstiegs von Armut und sozialer Härten sehr gravierend. Aiginger (2014a) weist darauf hin, dass wesentliche Ziele von „Europa 2020“ mittlerweile in fast unerreichbare Ferne gerückt sind. Gleichzeitig ist das eigentliche Ziel der Austeritätspolitik - die Senkung der Schuldenstandsquote - aufgrund der durch diese herbeigeführten wirtschaftlichen Schwäche verfehlt worden.

\section{Die jüngsten Pläne der EU-Kommission reichen nicht aus}

Seit knapp einem Jahr werden überall im Euroraum die Forderungen nach einer expansiveren Finanzpolitik lauter. Angesichts der drohenden deflationären Stagnation wird immer deutlicher, dass die Geldpolitik allein der Eurozone keinen Aufschwung wird bescheren können. In seiner mittlerweile berühmten - Rede in Jackson Hole hat EZBPräsident Mario Draghi eine expansivere Ausrichtung der Finanzpolitik in der Eurozone und ein öffentliches Investitionsprogramm gefordert, wobei dies aber im bestehenden institutionellen Rahmen zu geschehen habe (Draghi 2014). Der Europäische Rat sah bei seinem Treffen im Juni 2014 ebenfalls die Notwendigkeit einer Wachstumsstärkung. Gleichzeitig bestand er auf der Beibehaltung des bestehenden Regelwerkes: „Die Möglichkeiten, die der bestehende Haushaltsrahmen der EU bietet, um ein ausgewogenes Verhältnis zwischen Haushaltsdisziplin und notwendiger Unterstützung des Wachstums herzustellen, sollten genutzt werden“ (Europäischer Rat 2014, S. 7).

Unter Präsident Jean-Claude Juncker hat die EU-Kommission im Wesentlichen zwei Initiativen zur Wachstumsund Investitionsstärkung gestartet: Erstens die sogenannte Investitionsoffensive für Europa („Juncker-Plan“) und zweitens eine Klärung der Auslegung des SWP mit dem Ziel, Mitgliedstaaten in schwieriger Wirtschaftslage mehr fiskalischen Spielraum zu verschaffen.

Die erste Initiative strebt die Einrichtung eines Europäischen Fonds für Strategische Investitionen (EFSI) an, durch den - gemäß den Hoffnungen der Kommission - im Zeitraum von 2015 bis 2017 europaweit Investitionen in Höhe von $315 \mathrm{Mrd}$. $€$ angestoßen werden sollen (Europäische Kommission 2014b). Dies soll im Wesentlichen durch Umwidmung bestehender EU-Mittel in geringer Höhe erreicht werden, indem der bei der Europäischen Investitionsbank (EIB) angesiedelte EFSI mit 21 Mrd. $€$ Garantiekapital ausgestattet wird, von denen $16 \mathrm{Mrd}$. $€$ aus dem EU-Budget 
stammen und 5 Mrd. $€$ aus Reserven der EIB. Der Fond soll Kapital für Schlüsselinvestitionen in Infrastruktur, Bildung, Forschung und Innovation mobilisieren. Mit den zur Verfügung gestellten $21 \mathrm{Mrd}$ € soll durch 15-fache Hebelung mittels entsprechender Finanzinstrumente insgesamt das angestrebte Gesamtinvestitionsvolumen von $315 \mathrm{Mrd}$. $€$ erreicht werden. Gefördert werden sollen sowohl öffentliche als auch private Investoren oder Public-private-partnerships (PPP) - zum überwiegenden Teil für Großprojekte (240 Mrd. $€$ ) und zum geringeren Teil für Investitionen in kleinen und mittleren Unternehmen (75 Mrd. €). Ein noch größeres Investitionsvolumen wird in Aussicht gestellt, falls private oder öffentliche Investoren zusätzliches Kapital zur Aufstockung des Fonds beitragen. Zu diesem Zweck werden entsprechende Ausgaben der Mitgliedstaaten im Rahmen des SWP als unschädlich eingestuft (Europäische Kommission 2015, S. 6f.).

Angesichts ihres frühen Entwicklungsstadiums fällt eine Beurteilung der Investitionsoffensive schwer. Es gibt allerdings noch viele offene Fragen und es ist zu bezweifeln, dass die ehrgeizigen Ziele erreicht werden können (Horn et al. 2015, S. 7ff.; Feigl 2015). Zunächst hat es aufgrund der langwierigen politischen Entscheidungsprozesse fast ein Jahr (bis September 2015) gedauert, bis der Fonds überhaupt die ersten Projekte angehen konnte. Aber auch danach dürfte noch viel Zeit ins Land gehen, bis die überwiegend langfristig angelegten Großprojekte umgesetzt werden können.

Die größten Zweifel bestehen aber bezüglich der Frage, ob es überhaupt gelingen kann, ausreichend zusätzliche Investitionen zu mobilisieren: Das Hauptproblem bei den privaten Investitionen dürften die pessimistischen Erwartungen der Investoren sein, die sich kaum durch noch so günstige Finanzierungsbedingungen überwinden lassen dürften. Wenn dennoch private Investoren einsteigen, ist die Gefahr von Mitnahmeeffekten entsprechend groß. Gerade bei PPP-Projekten stellt sich die Frage nach der Effizienz: Wenn den privaten Investoren günstige Renditen zugesichert werden, so müssen diese letztlich von den Steuerzahlern oder den zukünftigen Nutzern über Gebühren finanziert werden. Wenn der Fonds dagegen öffentliche Investitionen finanzieren soll, stellt sich die Frage, warum dies nicht regulär über die öffentlichen Haushalte abgewickelt wird. Wenn es sich um eine Umgehung des nationalen oder europäischen fiskalischen Regelwerkes handelt, könnte man auch gleich das Regelwerk anpassen. Insgesamt könnte der Juncker-Plan daher - trotz der unverkennbar guten Absichten - zu einer großen Enttäuschung werden.

Die zweite Initiative der Kommission betrifft die Klarstellung der zukünftigen Anwendung des SWP (Europäische Kommission 2015, S. 9ff.). Hierbei wird erstens die sogenannte Investitionsklausel weiter gefasst, sodass in Zukunft möglicherweise mehr Staaten davon profitieren können. Demnach können Ausgaben für die von der EU kofinanzierten Investitionsprojekte - im Rahmen der Struktur- und Kohäsionsfonds, der transeuropäischen Netze, aber auch des neu geschaffenen EFSI - kurzfristige Abweichungen vom mittelfristigen Haushaltsziel oder dem entsprechenden Anpassungspfad im Rahmen der präventiven Komponente des SWPs rechtfertigen. Allerdings kann die Regelung nur in Anspruch genommen werden, wenn sich der betroffene Mitgliedstaat in einer Rezession oder wirtschaftlichen Schwächephase befindet. Es darf darüber hinaus nicht zu einem übermäßigen Defizit kommen und die Abweichung muss noch während der Laufzeit des Stabilitäts- oder Konvergenzprogramms wieder kompensiert werden (ebd.).

Zweitens können bedeutende Strukturreformen im Rahmen der präventiven Komponente des SWP vorübergehende Abweichungen vom mittelfristigen Haushaltsziel oder dem Anpassungspfad dorthin rechtfertigen:

"Die Kommission trägt den positiven Auswirkungen von Strukturreformen auf die öffentlichen Finanzen im Rahmen der präventiven Komponente des Pakts Rechnung, sofern diese Reformen i) einen größeren Umfang haben, ii) nachprüfbare, direkte, langfristige positive Auswirkungen auf den Haushalt haben, unter anderem durch eine Steigerung des nachhaltigen Potenzialwachstums, und iii) vollständig umgesetzt werden“ (Europäische Kommission 2015, S. 13).

Auch im Rahmen der korrektiven Komponente des SWP können Strukturreformen als „relevanter Faktor” eingestuft werden, was zur Nichteröffnung eines Defizitverfahrens oder zur Einräumung einer längeren Frist für die Korrektur eines übermäßigen Defizits führen kann.

Drittens wurden Bestimmung und Bedeutung der Konjunkturlage spezifiziert. Im Rahmen der präventiven Komponente kann eine ungünstige Konjunkturlage zu einer Verringerung des erforderlichen Konsolidierungsvolumens auf dem Weg zum mittelfristigen Haushaltsziel führen. Bei außergewöhnlich schlechter Konjunktur kann die Konsolidierung ganz ausgesetzt werden; bei sehr schlechter Konjunktur kann sie auf 0,25\% des BIP reduziert werden, während zuvor ein Standarderfordernis von 0,5\% bestand. Im Rahmen der korrektiven Komponente wird die Messung der diskretionären Konsolidierungsanstrengungen verbessert, indem das bisherige, auf den strukturellen Budgetsaldo abstellende und stark prozyklische Verfahren durch ein stabileres ergänzt wird.

Viertens hat die Kommission verdeutlicht, dass eine schwere Rezession in der Eurozone oder der EU insgesamt für alle Mitgliedstaaten eine vorübergehende Abweichung vom Konsolidierungspfad sowohl im Rahmen der präventiven als auch der korrektiven Komponente rechtfertigen kann.

\section{Für eine expansivere Finanzpolitik im bestehenden institutionellen Rahmen}

Insgesamt verringert die Auslegung des SWP durch die neue EU-Kommission spürbar den Konsolidierungsdruck 
in der Eurozone. Sie ermöglicht damit jedoch lediglich eine verminderte Restriktion, nicht aber einen deutlich positiven fiskalpolitischen Impuls. Ein solcher wäre aber angesichts der weiterhin prekären Wirtschaftslage am Rande einer deflationären Stagnation notwendig. Eine weitreichendere Interpretation der Spielräume im Rahmen des bestehenden fiskalpolitischen Regelwerks kann jedoch hervorragend an die neue Auslegung durch die EU-Kommission anknüpfen. ${ }^{1}$ Dadurch könnte sicherlich ein echter positiver Fiskalimpuls von z. B. 1 bis $2 \%$ des BIP über zwei bis drei Jahre ermöglicht werden.

Übersicht 1 gibt einen Überblick über die im Folgenden skizzierten sieben Optionen. Zunächst könnte die Investitionsklausel weiter ausgelegt werden, z. B. indem sämtliche von der EU kofinanzierten temporären Investitionsprojekte ohne weitere restriktive Bedingungen vom SWP ausgenommen werden (1). Darüber hinaus könnten auch andere temporäre Investitionsprojekte analog zum Vorgehen der Kommission beim EFSI ausgenommen werden (2). Andernfalls stellt sich die Frage, warum die Kommission bestimmte Projekte begünstigt, wenn sie über den EFSI finanziert werden, nicht aber, wenn sie als reguläre öffentliche Investitionen der Mitgliedstaaten durchgeführt werden. Bestimmte zentrale Investitionsprojekte könnten zudem als Strukturreform interpretiert werden und so eine vorübergehende Abweichung vom Konsolidierungspfad begründen (3). Generell kann eine Ausnahme öffentlicher (Netto-)Investitionen vom SWP im Sinne der sogenannten "Goldenen Regel“ sinnvoll sein, da diese kurz- wie langfristig wachstumssteigernde Effekte haben und dadurch auch zukünftigen Generationen zugutekommen (ausführlich siehe Truger 2015a). Bei der Haushaltsanalyse durch die Mitgliedstaaten und die Kommission sollten zudem realistische Investitionsmultiplikatoren in einer Größenordnung von deutlich über Eins angesetzt werden: Zusätzliche öffentliche Investitionen finanzieren sich in diesem Fall zu einem erheblichen Anteil selbst, weshalb sie, zumindest im Rahmen des Defizitverfahrens, (annähernd) irrelevant sein könnten (4).

\section{ÜBERSICHT 1}

\section{Optionen für eine expansive Finanzpolitik in der EU}

\begin{tabular}{l}
\hline (1) aktiverer Einsatz der „Investitionsklausel“ \\
\hline (2) befristete Investitionsprogramme zulassen (analog zum EFSI) \\
\hline (3) Interpretation befristeter Investitionsprogramme als Strukturreform \\
\hline (4) Ansatz eines realistischen Investitionsmultiplikators bei der Haushaltsanalyse \\
\hline (5) Spielraum in ökonomisch schlechten Zeiten nutzen \\
\hline (6) Ausnahme für schwerwiegenden Abschwung in EU oder Eurozone nutzen \\
\hline (7) Einsatz besserer Konjunkturbereinigungsverfahren
\end{tabular}

Quelle: Zusammenstellung des Autors auf Basis von Europäische Kommission (2015).
Darüber hinaus sollte der Spielraum für eine expansivere Finanzpolitik ganz allgemein auch unabhängig von den klassischen öffentlichen Investitionen genutzt werden. Dies kann einmal unter Hinweis auf die schlechte Konjunktur in einzelnen Mitgliedstaaten (5), vor allem aber unter Inanspruchnahme der Ausnahmeregel bei einer außergewöhnlichen Rezession im Euroraum oder der EU als Ganzer (6) geschehen. In der gegenwärtigen Lage wäre insbesondere Letzteres tatsächlich angebracht. Die Kommission könnte analog zum European Economic Recovery Plan (EERP) aus dem Jahr 2008 ein Europäisches Anti-Krisenprogramm auflegen. Tatsächlich hat die EU-Kommission zur Illustration der Ausnahmeregel explizit den Vergleich mit dem EERP gewählt (Europäische Kommission 2015, S. 17). Als Bedingung für die Inanspruchnahme soll sie auf außergewöhnliche Situationen beschränkt bleiben. Die gegenwärtige prekäre Lage mit Deflationsgefahren, der schwersten ökonomischen Krise der Mitgliedstaaten seit dem Zweiten Weltkrieg und einer bereits an der Nullzinsgrenze operierenden Geldpolitik böte gewiss eine Möglichkeit zur Rechtfertigung einer expansiveren Finanzpolitik. Das dadurch realisierte Anti-Krisenprogramm könnte sich auf traditionelle öffentliche Investitionen beziehen. Alternativ oder zusätzlich könnten aber auch schnell umsetzbare Ausgaben für Bildung, Kinderbetreuung oder generell Ausgaben im Hinblick auf die gegenwärtig vernachlässigten Ziele der Europa-2020-Strategie, wie etwa soziale Inklusion, die besonders unter der Austeritätspolitik gelitten haben, getätigt werden (Aiginger 2014b).

Unterstützt würden sämtliche Optionen für eine expansive Finanzpolitik noch, wenn die EU-Kommissionsmethode der Konjunkturbereinigung, die bei der Haushaltsüberwachung eine so große Rolle spielt, überarbeitet würde (7). ${ }^{2}$ Wie in Abschnitt 4 beschrieben, besteht das Hauptproblem bei der Konjunkturbereinigung darin, dass eine konjunkturelle Krise/ein Boom schon nach relativ kurzer Zeit zu einem erheblichen Teil als strukturelle Krise/Boom interpretiert wird. Das bedeutet gleichzeitig, dass der Beitrag der Konjunktur zur aktuellen Haushaltslage unterschätzt wird, während ein wesentlicher Teil der Haushaltslage als strukturell eingeschätzt wird. Tabelle 1 verdeutlicht dies durch einen Vergleich der von der EU-Kommission im Frühjahr 2014 für das Jahr 2014 geschätzten Produktionslücke, d. h. der Abweichung der Wirtschaftslage von der Normallage, und dem strukturellen Budgetsaldo mit modifizierten Werten für zwölf Mitgliedsländer der Eurozone. Die Modifikation wurde so vorgenommen, dass die Entwicklung der Normallage, d. h. des potenziellen BIP-Wachstums, seit der Prognose der EU-Kommission im Frühjahr 2010, also vor

1 Vgl. Micossi/Peirce (2014) sowieTruger (2015b) zu früheren Überlegungen zur Auslotung bestehender Spielräume.

2 Vgl. hierzu ausführlicherTruger $(2014,2015 b)$ und die dort angegebene Literatur. 
TABELLE 1

\section{Produktionslücke, struktureller Budgetsaldo und mittelfristige Haushaltsziele}

Angaben in Prozent des BIP*

\begin{tabular}{|c|c|c|c|c|c|}
\hline & $\begin{array}{l}\text { Produktions- } \\
\text { lücke } 2014 \\
\text { (EK Fj. 2014) }\end{array}$ & $\begin{array}{l}\text { Produktions- } \\
\text { lücke } 2014 \\
\text { (modifiziert) }\end{array}$ & $\begin{array}{c}\text { Struktureller } \\
\text { Budgetsaldo } 2014 \\
\text { (EK Fj. 2014) }\end{array}$ & $\begin{array}{c}\text { Struktureller } \\
\text { Budgetsaldo } 2014 \\
\text { (modifiziert) }\end{array}$ & $\begin{array}{c}\text { Mittelfristiges } \\
\text { Haushaltsziel }\end{array}$ \\
\hline Eurozone (12 Länder) & $-2,7$ & $-5,7$ & $-1,1$ & 0,4 & $\left.-0,2^{1}\right)$ \\
\hline Belgien & $-1,1$ & $-2,3$ & $-2,3$ & $-1,5$ & 0,75 \\
\hline Deutschland & $-0,7$ & 0,2 & 0,5 & 0,0 & $-0,5$ \\
\hline Irland & $-1,0$ & $-10,5$ & $-4,5$ & $-0,7$ & 0 \\
\hline Griechenland & $-9,3$ & $-25,8$ & 1,0 & 9,1 & \\
\hline Spanien & $-6,7$ & $-10,5$ & $-2,4$ & $-0,8$ & 0 \\
\hline Frankreich & $-2,8$ & $-5,7$ & $-2,3$ & $-0,8$ & 0 \\
\hline Italien & $-3,6$ & $-8,7$ & $-0,8$ & 1,9 & 0 \\
\hline Luxemburg & $-1,6$ & $-12,0$ & 0,6 & 5,7 & 0,5 \\
\hline Niederlande & $-2,6$ & $-8,2$ & $-1,3$ & 2,0 & $-0,5$ \\
\hline Österreich & $-0,8$ & $-2,8$ & $-1,2$ & $-0,3$ & $-0,45$ \\
\hline Portugal & $-4,0$ & $-6,6$ & & & $-0,5$ \\
\hline Finnland & $-2,6$ & $-8,8$ & $-0,9$ & 2,1 & $-0,5$ \\
\hline
\end{tabular}

*gewichtetes Mittel der verfügbaren Einzelwerte für die angeführten Länder ohne Griechenland.

Quelle: Berechnungen des Autors auf Basis von Europäische Kommission (2010, 2014c, 2014d).

Einsetzen der starken Austerität, unverändert blieb. Es zeigt sich, dass die negative Produktionslücke in allen Ländern mit Ausnahme Deutschlands ohne die austeritätsbedingte Anpassung des potenziellen BIPs viel größer gewesen wäre als von der EU-Kommission 2014 ausgewiesen. Im Extremfall hätte sie in Griechenland bei knapp - $26 \%$ gelegen, was angesichts eines Einbruchs der Wirtschaftsleistung um $25 \%$ sicherlich plausibler ist als der von der EU-Kommission angenommene Wert von - 9,3\%, der letztlich mehrere Jahre ein erheblich negatives Potenzialwachstum annehmen muss.

Die erhebliche Verminderung der negativen Produktionslücke führt automatisch zu einer Erhöhung des strukturellen Defizits, weil nun ein bedeutender Teil der schlechten Haushaltslage nicht mehr als konjunkturbedingt, sondern als strukturell interpretiert wird, was im Rahmen des SWP (fast) unmittelbar zu einem höheren Konsolidierungsbedarf führt. Im Rahmen der präventiven Komponente sollen die Mitgliedstaaten möglichst zügig ihr mittelfristiges Haushaltsziel, das als Zielwert für das strukturelle Defizit fungiert, erreichen. Führt die austeritätsbedingte Verschlechterung der Konjunktur aber, wie erläutert, zu einer Erhöhung des strukturellen Defizits, vergrößert sich der Abstand zum mittelfristigen Haushaltsziel und mithin der Konsolidierungsbedarf. Wären die Schätzungen des Potenzialwachstums seit dem Frühjahr 2010 nicht angepasst worden, hätte das allen Mitgliedstaaten im Rahmen der präventiven Komponente erhebliche Spielräume eröffnet (Tabelle 1): Italien, Luxemburg, die Niederlande, Österreich und Finnland hätten ihr mittelfristiges Haushaltsziel schon
2014 erreicht und hätten Spielraum für expansive Maßnahmen. Schließlich würden die stark negativen Produktionslücken auch einen dringenden fiskalpolitischen Handlungsbedarf anzeigen, der auch im Rahmen der Verfahren für ein übermäßiges Defizit relevant ist: Für Spanien wäre die außerordentliche Schwere der Wirtschaftskrise mit einer Produktionslücke von $-10,5 \%$ festgestellt worden, was die Argumentation für eine Verlängerung des Anpassungszeitraumes erleichtert hätte. Für die Eurozone insgesamt hätte die Produktionslücke $-5,7$ anstatt $-2,7 \%$ des BIP betragen. Es soll hier nicht behauptet werden, dass die modifizierten Werte aus Tabelle 1 die „wahren“ Werte darstellen. Sie sind lediglich illustrativ zu verstehen und verdeutlichen, welchen Spielraum eine weniger prozyklische Methode der Konjunkturbereinigung für die Mitgliedstaaten in der Krise ermöglichen würde. ${ }^{3}$

\section{Fazit}

Das Europa beherrschende makroökonomische Paradigma ist im Kern wachstums- und beschäftigungsschädlich

3 Es sei darauf hingewiesen, dass der SWP symmetrisch im Aufschwung entsprechend eine restriktivere Finanzpolitik erfordern würde. Genau dies ist im Sinne einer antizyklischen Politik auch erwünscht. 
und sozialstaatsfeindlich. Hierbei handelt es sich leider nicht nur um ein intellektuelles, sondern auch um ein ganz praktisches Problem: Dieses Paradigma prägt in weiten Teilen die wirtschaftspolitischen Institutionen und die tatsächliche Wirtschaftspolitik der EU und insbesondere der Währungsunion. Eine nachhaltige Lösung der Wirtschafts- und Beschäftigungskrise in der EU setzt daher mittel- bis langfristig eine Überwindung des herrschenden makroökonomischen Paradigmas im Sinne einer Revitalisierung des Keynesianismus und eine entsprechende Reform des europäischen Institutionengefüges im Sinne eines sozialen Europas voraus. Der Weg dahin kann kurzfristig über eine möglichst weitgehende Nutzung der existierenden Spielräume innerhalb des bestehenden Regelwerks begonnen werden. Ein Ende der Austeritätspolitik und eine expansive Fiskalpolitik können die Wirtschaftskrise beenden und zu mehr Wachstum und Beschäftigung und damit auch zu einer erheblichen Linderung der sozialen Misere führen.

Die EU-Kommission ist mit ihrer jüngsten Interpretation der europäischen Fiskalregeln bereits einen bedeutenden Schritt in Richtung der notwendigen Revitalisierung der nationalen Finanzpolitik als makroökonomischem Instrument gegangen. Damit hat sie die Austeritätspolitik nach einem langsamen - und für die europäische Bevölkerung extrem schmerzhaften pathologischen Lernprozess in ihrer schlimmsten Form hinter sich gelassen. Es bleibt zu hoffen, dass sie bald noch konsequentere Schritte für einen echten positiven fiskalischen Impuls gehen wird, der die Eurozone nach sieben Krisenjahren endlich wieder in einen selbsttragenden, kräftigen Aufschwung führt. Der vorliegende Beitrag hat gezeigt, dass die Möglichkeiten hierzu auch im bestehenden finanzpolitischen Rahmen zweifellos gegeben wären, wenn die EU-Kommission und der Europäische Rat zu einer entsprechenden Interpretation bereit wären. Eine Umkehr zum sozialen Europa ist möglich zur Not selbst innerhalb der bestehenden EU-Institutionen.

\section{LITERATUR}

Aiginger, K. (2014a): The Europe 2020 strategy at midterm: disappointing assessment calls for an urgent change driven by long run priorities. Response of the Austrian Institute of Economic Research (WIFO) to the public consultation on the Europe 2020 strategy: European Commission IP/14/50405/05/2014, Wien Aiginger, K. (2014b): A silver bullet for restarting growth in Europe, EurActiv. com PLC, http://www.euractiv.com/sections/euro-finance/silver-bullet-restarting growth-europe-310912

Arestis, P. (2011): Keynesian economics and the New Consensus in macroeconomics, in: Hein, E./Stockhammer, E. (Hrsg.): A modern guide to Keynesian macroeconomics and economic policies, Cheltenham

Arestis, P./Sawyer, M. (2003): Reinventing fiscal policy, in: Journal of Post Keynesian Economics 26 (1), S. 3-25

Baker, D./Glyn, A./Howell, D./Schmitt, J. (2004): Labour market institutions and unemployment: a critical assessment of the cross-country evidence, in: Howell, D. R. (Hrsg.): Fighting unemployment. The limits of the free market orthodoxy, Oxford, S. 72-117

Blyth, M. (2013): Austerity. The history of a dangerous idea, Oxford

Carnot, N./de Castro, F. (2015): The discretionary fiscal effort: an assessment of fiscal policy and its output effect: European Commission, Economic Papers (543), Brussels

Darvas, Z./HuettI, P./de Sousa, C./Terzi, A./Tschekassin, O. (2014): Austerity and poverty in the European Union: European Parliament, Study for the Committee on Employment and Social Affairs, Brussels

Draghi, M. (2014): Unemployment in the euro area, Speech at the Federal Reserve Bank of Kansas City Economic Symposium, Jackson Hole, Wyoming 22 August, http://www.bis.org/review/r140826a.pdf

Europäische Kommission (2010): Annual macro-economic database (Ameco), May

Europäische Kommission (2013): Building a strengthened fiscal framework in the European Union: a guide to the Stability and Growth Pact, European Economy, Occasional Papers (150), Brüssel
Europäische Kommission (2014a): Annual macro-economic database, November, Brüssel

Europäische Kommission (2014b): Mitteilung der Kommission an das Europäische Parlament, den Rat, die Europäische Zentralbank, den Wirtschafts- und Sozialausschuss, den Ausschuss der Regionen und die Europäische Investititonsbank: Eine Investitionsoffensive für Europa, 26.11., COM(2014) 903 final/2, Brüssel

Europäische Kommission (2014c): The 2014 stability and convergence programmes: an overview: European Commission, Directorate-General for Economic and Financial Affairs, Occasional Papers (199), Brüssel

Europäische Kommission (2014d): Annual macro-economic database (Ameco) Spring, Brüssel

Europäische Kommission (2015): Mitteilung der Kommission an das Europäische Parlament, den Rat, die Europäische Zentralbank, den Wirtschafts- und Sozialausschuss, den Ausschuss der Regionen und die Europäische Investitionsbank: Optimale Nutzung der im Stabilitätspakt vorgesehenen Flexibilität, Straßburg, 13.1. COM(2015) 12 final, Brüssel

Europäischer Rat (2014): Tagung vom 26./27. Juni, Schlussfolgerungen, EUCO 79/14, CO EUR 4 CONCL 2, Brüssel

Feigl, G. (2015): Investitions- und Ausgabenmöglichkeiten unzureichend gelockert: Kammer für Arbeiter und Angestellte, Infobrief EU und International (1), Wien Gechert, S. (2015): Are fiscal multipliers regime-dependent? A meta regression analysis, in: Oxford Economic Papers 67 (4), S. 865-888

Heimberger, P. (2014): Das strukturelle Defizit: Methodische Probleme und politische Implikationen, in: Momentum Quarterly: Zeitschrift für Sozialen Fortschritt 3 (3), S. 125-148

Hein, E./Niechoj, T. (2007): Guidelines for sustained growth in the EU? The concept and the consequences of the Broad Economic Policy Guidelines, in: McCombie, J./Rodriguez, C. (Hrsg.):The European Union. Current problems and prospects, Basingstoke

Hein, E./Truger, A. (2004): Makroökonomische Koordinierung als wirtschaftspolitisches Konzept. Voraussetzungen und Möglichkeiten in der EU, in: Hein, E./Niechoj, T./Schulten,Th./Truger, A. (Hrsg.): Europas Wirtschaft gestalten. Makroökonomische Koordinierung und die Rolle der Gewerkschaften, Hamburg, S. 15-50

Hein, E./Truger, A. (2007): Fiscal policy and macroeconomic performance in the Euro area: lessons for the future, in: Bibow, J./Terzi, A. (Hrsg.): Euroland and the world economy. Global player or global drag?, Basingstoke,

S. $154-183$

Hein, E./Stockhammer, E. (2011): A post-Keynesian macroeconomic model of inflation, distribution and employment, in: Hein, E./Stockhammer, E. (Hrsg.): A modern guide to Keynesian macroeconomics and economic policies, Cheltenham

Hein, E./Truger, A./van Treeck, T. (2012): The European financial and economic crisis: alternative solutions from a (Post-)Keynesian perspective, in: Arestis, P./Sawyer, M. (Hrsg.): The euro crisis, Basingstoke, S. 35-78

Hein, E./Niechoj, T./Schulten, Th./Truger, A. (Hrsg.) (2004): Europas Wirtschaft gestalten. Makroökonomische Koordinierung und die Rolle der Gewerkschaften, Hamburg

Heine, M./Herr, H. (2013): Volkswirtschaftslehre, 4. Aufl., München Hermann, C./Hinrichs, K. (2012): Die Finanzkrise und ihre Auswirkungen auf Sozialstaaten und Arbeitsbeziehungen - ein europäischer Rundblick: Forschungs- und Beratungsstelle Arbeitswelt, Projektbericht, Wien

Horn, G./Gechert, S./Herzog-Stein, A./Rannenberg, A./Rietzler, K./Tober, S. (2015): Wirtschaftspolitische Herausforderungen 2015: Wirtschaftspolitik unter Zwängen: Institut für Makroökonomie und Konjunkturforschung in der Hans-BöcklerStiftung, IMK Report (102), Düsseldorf

Issing, O. (2002): On macroeconomic policy co-ordination in EMU, in: Journal of Common Market Studies 40 (2), S. 354-358

Klär, E. (2014): Die Eurokrise im Spiegel der Potenzialschätzungen: Lehren für eine alternative Wirtschaftspolitik?: Friedrich-Ebert-Stiftung, WiSo-Diskurs April, Bonn Lavoie, M. (2009): Taming the New Consensus: hysteresis and some other Post-Keynesian amendments, in: Fontana, G./Setterfield, M. (Hrsg.): Macroeconomics and macroeconomic pedagogy, Basingstoke Micossi, S./Peirce, F. (2014): Flexibility clauses in the Stability and Growth Pact: no need for revision: Centre for European Policy Studies, CEPS Policy Brief (319), Brussels

OECD (Organisation for Economic Co-operation and Development) (1994):

The OECD Jobs Study: evidence and explanations, Paris

Schulmeister, S. (2010): Mitten in der großen Krise. Ein "New Deal” für Europa, Wien 
Snowdon, B./Vane, H. (2005): Modern macroeconomics. Its origin, development and current state, Cheltenham

Truger, A. (2003): Der missachtete Mainstream - Die deutsche wirtschaftspolitische Debatte leidet weniger unter dem Neu-Keynesianismus als unter seiner fast völligen Missachtung, in: Hein, E./Heise, A./Truger, A. (Hrsg.): Neu-Keynesianismus - der neue wirtschaftspolitische Mainstream? Marburg, S. 245-278 Truger, A. (2013): Austerity in the euro area: the sad state of economic policy in Germany and the EU, in: European Journal of Economics and Economic Policies: Intervention 2013 (2), S. 158-174

Truger, A. (2014): Mehr Glück als Verstand: Die deutsche Finanzpolitik ist kein Vorbild für Europa, in: Junkernheinrich, M./Korioth, S./Lenk, T./Scheller,

H./Woisin, M. (Hrsg.): Jahrbuch für öffentliche Finanzen 2014, Berlin, S. 279-297 Truger, A. (2015a): Implementing the golden rule for public investment in Europe: safeguarding public investment and supporting the recovery: Abteilung Wirtschaftswissenschaft und Statistik der Kammer für Arbeiter und Angestellte Wien, Materialien zu Wirtschaft und Gesellschaft (138), Wien

Truger, A. (2015b): The fiscal compact, cyclical adjustment and the remaining leeway for expansionary fiscal policies in the Euro area, in: Panoeconomicus $62(2)$, S. 157-175

Truger, A./Hein, E. (2003): "Schlusslicht Deutschland" - Resultat institutioneller Verkrustungen?, in: Wirtschaftsdienst. Zeitschrift für Wirtschaftspolitik 83 (8), S. $509-516$
Truger, A./Will, H. (2012): Gestaltungsanfällig und pro-zyklisch: Die deutsche Schuldenbremse in der Detailanalyse, in: Hetschko, Cl./Pinkl, J./Pünder, H./Thye, M. (Hrsg.): Staatsverschuldung in Deutschland nach der Föderalismusreform II - Eine Zwischenbilanz, Hamburg, S. 75-100

\section{AUTOR}

ACHIM TRUGER, Dr. rer pol., ist Professor für Volkswirtschaftslehre, insbesondere Makroökonomie und Wirtschaftspolitik, und Prodekan des Fachbereichs Wirtschaftswissenschaften der Hochschule für Wirtschaft und Recht (HWR) in Berlin sowie Senior Research Fellow am Institut für Makroökonomie und Konjunkturforschung (IMK) in der Hans-Böckler-Stiftung in Düsseldorf. Arbeitsschwerpunkte: Makroökonomie und Wirtschaftspolitik, Steuer- und Finanzpolitik.

achim.truger@hwr-berlin.de 